\title{
ФИКСИРОВАННЫЕ ИДЕАЛЫ В ТОПОЛОГИЧЕСКИХ МОДУЛЬ-АЛГЕБРАХ
}

\author{
(Представил А. Хумал)
}

1. Пусть $\mathbf{F}$ - поле $\mathbf{R}$ или $\mathbf{C}$ и $A$ - топологическая $\mathbf{F}$-алгебра, т. е. такое линейное топологическое пространство над $\mathrm{F}$, которое является ассоциативной алгеброй относительно определенного на $A$ умножения элементов, и в котором умножение элементов (как билинейное отображение $A \times A$ в $A)$ раздельно непрерывно. В частности, когда умножение элементов непрерывно в совокупности, A называется топологической F-алгеброй с непрерывным умножением. При этом $A$ называется локально выпуклой (локально ограниченной, локально псевдовыпуклой и т. д.) $\mathrm{F}$-алгеброй, если она (как линейное топологическое пространство) локально выпукла (соответственно локально ограничена, локально псевдовыпукла * и т. д.).

Пусть теперь $A$ - топологическая $\mathbf{F}$-алгебра с единицей (здесь и всюду в дальнейшем через $e_{A}$ будем обозначать единицу в $A$ ) и $\operatorname{Inv} A$ - множество всех обратимых элементов в $A$. Если в $A$ существует окрестность единицы, все элементы которой обратимы в $A$, и обращение в $A$ (как отображение $\operatorname{Inv} A$ в $\operatorname{Inv} A$ ) непрерывно в точке $e_{A}$, то $A$ называется топологической F-алгеброй с непрерывным обратным. Известно (см., напр., $\left[{ }^{1}\right]$, с. 205), что при топологической $\mathrm{F}$-алгебре $A$ с непрерывным обратным множество Inv $A$ открыто в топологии алгебры $A$ и операция $a \rightarrow a^{-1}$ непрерывна на Inv $A$.

Рассмотрим теперь случай, когда $A$ является локально выпуклой $\mathrm{F}$-алгеброй. Пусть $\left\{p_{\lambda}: \lambda \in \Lambda\right\}-$ система полунорм на $A$, определяющая ее топологию. Если все полунормы $p_{\lambda}$ на $A$ удовлетворяют условию $p_{\lambda}(a b) \leqslant p_{\lambda}(a) p_{\lambda}(b)$ при всех $a, b \in A$, то $A$ называется локально $m$-выпуклой F-алгеброй, а если для каждых $\lambda \in \Lambda$ и $a_{0} \in A$ существуют положительные числа $M_{\lambda}\left(a_{0}\right)$ и $N_{\lambda}\left(a_{0}\right)$ такие, что $p_{\lambda}\left(a_{0} a\right) \leqslant M_{\lambda}\left(a_{0}\right) p_{\lambda}(a)$ и $p_{\lambda}\left(a a_{0}\right) \leqslant N_{\lambda}\left(a_{0}\right) p_{\lambda}(a)$ при всех $a \in A$, то $A$ называется поглощенно выпуклой или $A$-выпуклой F-алгеброй. Таким образом, локально $m$-выпуклые F-алгебры поглощенно выпуклы, но существуют поглощенно выпуклые F-алгебры, которые не являются локально $m$-выпуклыми (см. $\left[{ }^{2}\right]$, с. 20 и $\left[{ }^{3}\right]$, с. 155$)$. Кроме того, в $\left[{ }^{3}\right]$ на с. 154 и в $\left[{ }^{4}\right]$ на с. 87 приведены примеры таких локально $m$-выпуклых F-алгебр с единицей и в $\left[{ }^{4}\right]$ на с. 87 - таких полных метризуемых локально выпуклых F-алгебр с единицей, в которых множество Inv $A$ не является открытым. Учитывая

* Линейное топологическое пространство называется .өкально псевдовыпуклым, если оно имеет базу окрестностей нуля, состоящую из псевдовыпуклых подмножеств, т. е. подмножеств $U$, удовлетворяющих следующим условиям: $U+U \subseteq \lambda U$ при некотором числе $\lambda>0$ и $\mu U \subseteq U$ при всех $\mu$ с $|\mu| \leqslant 1$. Нетрудно заметить, что каждое локально выпуклое и каждое локально ограниченное пространство локально псездовыпукло. 
в добавок и то, что поглощенно выпуклые F-алгебры не всегда метризуемы и полны, а полные метризуемые локально выпуклые F-алгебры не всегда поглощенно выпуклы, становится очевидным, что поглощенно выпуклые F-алгебры с единицей, полные метризуемые локально выпуклые F-алгебры с единицей и локально псевдовыпуклые $\mathbf{F}$-алгебры с непрерывным обратным образуют различные классы топологических F-алгебр.

Топологическая F-алгебра $B$ называется (см. $\left.\left[{ }^{5}\right]\right)$ топологической модуль-алгеброй относительно $А$ или, коротко, топологической $(A, \mathbf{F})$-алгеброй, если

а) А есть топологическая F-алгебра,

б) В есть А-бимодуль,

в) модульные умножения в $B$ (т. е. отображений $(a, b) \rightarrow a b$ и $(b, a) \rightarrow b a$ произведений $A \times B$ в $B$ и $B \times A$ в $B)$ раздельно непрерывны и

г) справедливы $a\left(b_{1} b_{2}\right)=\left(a b_{1}\right) b_{2}, \quad\left(b_{1} b_{2}\right) a=b_{1}\left(b_{2} a\right)$ и $\left(b_{1} a\right) b_{2}=$ $=b_{1}\left(a b_{2}\right)$ при всех $a \in A u b_{1}, b_{2} \in B$.

Кроме того, если $A$ имеет единицу, то $e_{A} b=b=b e_{A}$ для всех $b \in B$, а если $B$ имеет единицу, то $e_{B} a=a e_{B}$ для всех $a \in A$. Топологическая $(A, \mathrm{~F})$-алгебра называется топологической $(A, \mathrm{~F})$-алгеброй с непрерывными модульными умножениями, если модульные умножения в $B$ непрерывны в совокупности.

2. Пусть $A$ - отделимая топологическая $\mathrm{F}$-алгебра, $B$ - топологическая $(A, \mathbf{F})$-алгебра, $\operatorname{hom}(B, A)-$ множество всех нетривиальных непрерывных $A$-гомоморфизмов $B$ на $A$ (т. е. гомоморфизмов $Ф$ алгебры $B$ на $A$, удовлетворяющих условиям $\Phi(a b)=a \Phi(b)$ и $\Phi(b a)=\Phi(b) a$ при всех $a \in A$ и $b \in B)$ и hom $B=\operatorname{hom}(B, \mathbf{F})$. Множество hom $(B, A)$ наделим топологией, в которой предбазу окрестностей гомоморфизма $\Phi_{0} \in \operatorname{hom}(B, A)$ образуют множества

$$
\left\{\Phi \in \operatorname{hom}(B, A): \Phi(b)-\Phi_{0}(b) \in O\right\},
$$

где $b \in B$ и $O$ есть окрестность нуля в $A$.

Пусть теперь $B$ - такая топологическая $(A, \mathrm{~F})$-алгебра, которая содержит $\mathbf{F}$-подалгебру $Z$, множество hom $Z$ которой непусто (в $\left[{ }^{3,4,6}\right.$ ] приведены примеры топологических $\mathbf{F}$-алгебр $A$, множество hom $A$ которых пусто) и удовлетворяет условиям

(a) A-подмодуль, порожденный F-подалгеброй $Z$, всюду плотен в $B$ и

(б) для каждого $\varphi \in \operatorname{hom} Z$ существует $\Phi_{\varphi} \in \operatorname{hom}(B, A)$ такой, что $\Phi_{\varphi}(a z)=\Phi_{\varphi}(z a)=a \varphi(z)$ при всех $a \in A u z \in Z$. вию

Нетрудно заметить, что условие (б) является равносильным усло-

(б') для каждого $\varphi \in$ hom $Z$ существует $\Phi_{\varphi} \in \operatorname{hom}(B, A)$ такой, что $\Phi_{\varphi}(z)=e_{A} \varphi(z)$ при всех $z \in Z$,

если $A$ имеет единицу. По условию (а) каждый $\varphi \in \operatorname{hom} Z$ определяет гомоморфизм $\Phi_{\varphi}$ единственным образом (ввиду отделимости топологии алгебры $A$ ). Учитывая это, для каждого фиксированного элемента $b \in B$ жижогош $b^{\wedge}(\varphi)=\Phi_{\varphi}(b)$ при всех $\varphi \in \operatorname{hom} Z$ и $\mathcal{t}(b)=b^{\wedge}$. Тогда $b^{\wedge}$ отображает hom $Z$ в A, а $\mathcal{A}$ отображает $B$ в $A^{\text {homz }}$.

Левый (правый и двусторонний) идеал $J$ топологической $(A, \mathbf{F})$-алгебры $B$, удовлетворяющей условиям (а) и (б), будем называть фиксированным, если существует $\varphi \in \operatorname{hom} Z$ (по условию (б) и $\Phi_{\varphi} \in$ $\in \operatorname{hom}(B, A))$ такой, что множество $\Phi_{\varphi}(\mathfrak{J})$ принадлежит некоторому левому (соответственно правому и двустороннему) идеалу в $A$. Идеалы в $B$, не являющиеся фиксированными, будем называть свободными.

В данной статье дается описание всех фиксированных регулярных 
максимальных идеалов (в частности, замкнутых максимальных й тех максимальных идеалов, коразмерность которых равна единице) в топологических модуль-алгебрах, и определяются классы топологических модуль-алгебр, в которых фиксированы

а) все замкнутые регулярные максимальные идеалы коразмерности один,

б) все замкнутые максимальные идеалы и

г) все идеалы.

\section{§ 1. Описание фиксированных регулярных максимальных идеалов}

Пусть $A$ - топологическая $\mathbf{F}$-алгебра, $\mathfrak{M}_{l}(A)$ и $\mathfrak{M}_{r}(A)-$ множества всех регулярных максимальных (соответственно левых и правых) идеалов в $A, \mathfrak{M}_{1}(A)$ - подмножество тех идеалов в $\mathfrak{M}(A)=\mathfrak{M}_{l}(A) \cap \mathfrak{M}_{r}(A)$, коразмерность которых равна единице, $\mathfrak{M}_{k c}(A)$ с $k=l, r, 1$ и $\mathfrak{M}_{c}(A)-$ подмножества замкнутых идеалов в $\mathfrak{M}_{k}(A)$ и $\mathfrak{M}(A)$ соответственно. Пусть далее $\widetilde{\mho}_{k}(A)\left(\widetilde{\Im}_{k c}(A)\right)$ с $k=l, r, 1$ - подмножество фиксированных (соответственно замкнутых фиксированных) идеалов в $\mathfrak{M}_{k}(A)$ и, кроме того, $\mho(A)=\mho_{l}(A) \cap \mathfrak{\mho}_{r}(A)$ и $\mho_{c}(A)=\mathfrak{\mho}_{l c}(A) \cap \mathfrak{\mho}_{r c}(A)$.

Следующая теорема дает описание фиксированных регулярных максимальных идеалов в топологических модуль-алгебрах.

Те орем а 1. Пусть $A$ - отделимая топологическая F-алгебра и $B$ топологическая $(A, \mathrm{~F})$-алгебра, содержащая $\mathrm{F}$-подалгебру $Z$, множество hom $Z$ которой непусто. Если выполнены условия (а) и (б), то каждый идеал $\mathbb{H} \in \mathfrak{F}_{l}(B)$ определяет $\varphi \in \operatorname{hom} Z \quad$ и $M \in \mathfrak{M}_{l}(A)$ такие, что $\mathcal{M}=\Phi_{\varphi}^{-1}(M) \quad$ и отображение $(M, \varphi) \rightarrow \Phi_{\varphi}^{-1}(M)$ является биекцией $\mathfrak{M}_{l}(A) \times \operatorname{hom} Z$ на $\mathfrak{\mho}_{l}(B)$.

Аналогичные описания имеют место и для множеств $\mathfrak{F}_{k}(B), \mathfrak{\mho}_{s c}(B) u$ $\widetilde{\mho}(B)$, где $k=c, r, 1$ и $s=l, r, 1$.

Доказательство. а) Пусть $\mathscr{M} \in \mathfrak{F}_{l}(B)$ и $b_{0}-$ правая единица по идеалу $\mathfrak{M}$. Тогда по условию (б) существует гомоморфизм $\Phi_{\varphi} \in$ $\in \operatorname{hom}(B, A)$ при некотором $\varphi \in \operatorname{hom} Z$ такой, что $\Phi_{\varphi}(\mathcal{M})$ принадлежит нскоторый левый идеал в $A$. Поэтому $M=\Phi_{\varphi}(M)$ образует в $A$ регулярный левый идеал и $u \in \Phi_{\varphi}\left(b_{0}\right)$ является правой единицей по идеалу $M$.

Пусть теперь $I$ - любой левый идеал в $A$, содержащий $M$. Тогда $u$ есть правая единица и по идеалу $I$. Поэтому $u \notin I$ и $\Phi_{\varphi}^{-1}(I) \neq B$ (если это не так, то $\left.u \in \Phi_{\varphi}\left[\Phi_{\varphi}^{-1}(I)\right]=I\right)$. Значит, $\Phi_{\Phi}^{-1}(I)$ есть регулярный левый идеал в $B$, содержащий $\mathcal{M}$. В силу этого, $I=M$. Следовательно, $M \in \mathbb{M}_{l}(A)$ и $\mathscr{M}=\Phi_{\varphi}^{-1}(M)$.

Предположим, что кроме гомоморфизма ф существует еще гомоморфизм $\varphi^{\prime} \in \operatorname{hom} Z$ отличный от $\varphi$ такой, что $\Phi_{\varphi^{\prime}}(\mathcal{M}) \subset I^{\prime}$ для некоторого левого идеала $I^{\prime} \subset A$. Тогда $a \varphi^{\prime}\left(z_{0}\right)=\Phi_{\varphi^{\prime}}\left(a z_{0}\right) \in I^{\prime}$ для каждого $a \in A$ и $z_{0} \in \operatorname{ker} \varphi \backslash \operatorname{ker} \varphi^{\prime}$, ибо $a z_{0} \in \operatorname{ker} \Phi_{\varphi}$ и $\operatorname{ker} \Phi_{\varphi} \subset \Phi_{\varphi}^{-1}(M)=\mathcal{M}$. Кроме того, $u^{\prime}=\Phi_{\varphi^{\prime}}\left(b_{0}\right)$ есть правая единица по идеалу $I^{\prime}$. Поскольку $\varphi^{\prime}\left(z_{0}\right) \neq 0$, то из $u^{\prime} \varphi^{\prime}\left(z_{0}\right) \in I^{\prime}$ следует $u^{\prime} \in I^{\prime}$, что не возможно. Значит, каждый идеал $\mathbb{M} \in \mathfrak{F}_{l}(B)$ определяет единственным образом гомоморфизм $\varphi \in \operatorname{hom} Z$ такой, что $\mathcal{H}=\Phi_{\varphi}^{-1}\left[\Phi_{\varphi}(\mathcal{H})\right]$.

Пусть теперь $\varphi \in \operatorname{hom} Z, M \in \mathfrak{M}_{l}(A)$ и $u$ - правая единица по идеалу $M$. Тогда по условию (б) существует $\Phi_{\varphi} \in \operatorname{hom}(B, A)$. Пусть $\nVdash=\Phi_{\varphi}^{-1}(M)$ и $b_{0} \in B-$ такой, что $u=\Phi_{\varphi}\left(b_{0}\right)$. Тогда $M \neq B$ (в противном случае $u \in \Phi_{\varphi}\left[\Phi_{\varphi}^{-1}(M)\right]=M$, что не возможно). Поэтому $\mathcal{M}$ образует в $B$ регулярный левый идеал, и $b_{0}$ есть правая единица по идеалу $\mathcal{M}$. Пусть $\mathcal{L}$ - любой регулярный левый идеал в $B$, содержащий $\mathscr{M}$. Если 
$\tilde{\Phi}_{\varphi}(\mathfrak{L})=\dot{A}$, то $u=\Phi_{\varphi}\left(b^{\prime}\right)$ для некоторого элемента $b^{\prime} \in \mathcal{L}$, в силу чего̆ $\Phi_{\varphi}\left(b-b b^{\prime}\right) \in M$. Поэтому из $b-b b^{\prime} \in \mathcal{M} \subset \mathcal{L}$ следует, что $b=$ $=\left(b-b b^{\prime}\right)+b b^{\prime} \in \mathcal{L}$ при всех $b \in B$, но это не возможно. Таким образом, $\Phi_{\varphi}(\mathcal{L}) \neq A$. Учитывая это, $\Phi_{\varphi}(\mathcal{L})$ образует в $A$ регулярный левый идеал и содержит $M$. Следовательно, $\Phi_{\varphi}(\mathcal{L})=M$ ввиду максимальности идеала $M$ и $\mathcal{L}=\mathcal{M}$. Итак, $\mathcal{M} \in \mathbb{M}_{l}(B)$ и $\Phi_{\varphi}(\mathcal{M})=M$. Значит, $\mathcal{M} \in \mathfrak{F}_{l}(B)$.

Пусть далее $Y=m_{l}(A) \times$ hom $Z$. Чтобы показать инъективность отображения $(M, \varphi) \rightarrow \Phi_{\varphi}^{-1}(M)$ предположим, что $(M, \varphi), \quad\left(M^{\prime}, \varphi^{\prime}\right) \in Y$ и $(M, \varphi) \neq\left(M^{\prime}, \varphi^{\prime}\right)$. Тогда, либо $M \neq M^{\prime}$, либо $\varphi \neq \varphi^{\prime}$. Если $M \neq M^{\prime}$, то $z a \in \Phi_{\varphi}^{-1}(M) \backslash \Phi_{\varphi}^{-1}\left(M^{\prime}\right)$ при $a \in M \backslash M^{\prime}$ и $z \in Z \backslash\left(\operatorname{ker} \varphi \bigcup \operatorname{ker} \varphi^{\prime}\right)$ независимо от того, равны $\varphi$ и $\varphi^{\prime}$ или нет. Кроме того, если $\varphi \neq \varphi^{\prime}$, то ker $\varphi \neq$ $\neq \operatorname{ker} \varphi^{\prime} \quad$ (см. $\left[{ }^{7}\right], \quad$ с. 321). Поэтому $a z \in \Phi_{\varphi^{-1}}(M) \backslash \Phi_{\psi^{\prime}}-1\left(M^{\prime}\right)$ при $a \in A \backslash\left(M \cup M^{\prime}\right)$ и $z \in \operatorname{ker} \varphi \backslash \operatorname{ker} \varphi^{\prime}$, независимо от того, совпадают̃ идеалы $M$ и $M^{\prime}$ или нет. Таким образом, из $(M, \varphi) \neq\left(M^{\prime}, \varphi^{\prime}\right)$ следует $\Phi_{\varphi}^{-1}(M) \neq \Phi_{\varphi^{\prime}}-1\left(M^{\prime}\right)$. Значит, отображение $(M, \varphi) \rightarrow \Phi_{\varphi}^{-1}(M)$ является биекцией $Y$ на $\mathfrak{\mho}_{l}(B)$.

б) Пусть $\mathcal{M} \in \mathfrak{\mho}_{l c}(B)$. Тогда по вышедоказанному, существуют $\varphi \in$ $\in \operatorname{hom} Z$ и $M \in \mathfrak{M}_{l}(A)$ такие, что $\mathcal{M}=\Phi_{\varphi}{ }^{-1}(M)$. Чтобы показать замкнутость идеала $M$, берем любой элемент $a_{0}$ из замыкания идеала $M$. Тогда найдется сеть $\left(a_{\lambda}\right)_{\lambda \in \Lambda} \subset M$, которая сходится к $a_{0}$. Пусть далее $z_{0}-$ любой фиксированный элемент в $Z \backslash \operatorname{ker} \varphi$. Тогда из $a_{\lambda} \varphi\left(z_{0}\right) \in M$ при всех $\lambda \in \Lambda$ следует, что $\left(a_{\lambda} z_{0}\right)_{\lambda \in \Lambda} \subset \mathcal{H}$. В силу непрерывности отображения $a \rightarrow a z_{0}$ из сходимости сети $\left(a_{\lambda}\right)_{\lambda \in \Lambda}$ к $a_{0}$ следует сходимость сети $\left(a_{\lambda} z_{0}\right)_{\lambda \in \Lambda}$ к $a_{0} z_{0}$ и справедливо $a_{0} z_{0} \in \mathcal{M}$ ввиду замкнутости идеала $\mathcal{N}$. Поэтому из $a_{0 \varphi}\left(z_{0}\right)=\Phi_{\varphi}\left(a_{0} z_{0}\right) \in M$ следует, что $a_{0} \in M$, в силу чего, $M \in \mathbb{M}_{l c}(A)$. Кроме того, идеал $\Phi_{\varphi}^{-1}(M)$ является замкнутым в $B$ при всех $\varphi \in \operatorname{hom} Z$ и $M \in \mathbb{M}_{l c}(A)$ (ввиду непрерывности гомоморфизма $\left.\Phi_{\varphi}\right)$. Таким образом, $(M, \varphi) \rightarrow \Phi_{\varphi}^{-1}(M)$ отображает $\mathfrak{M}_{l c}(A) \times$ $X$ hom $Z$ взаимно однозначно на $\Im_{l c}(B)$.

в) Пусть $\mathcal{H} \in \mathfrak{\mho}_{1}(B)$. Тогда (опять по вышедоказанному) идеал $\mathcal{M}=\Phi_{\varphi}^{-1}(M)$ при некоторых $\varphi \in \operatorname{hom} Z$ и $M \in \mathfrak{M}(A)$. Пусть $\pi_{B}\left(\pi_{A}\right)-$ естественный гомоморфизм $B$ на $B / \mathcal{M}$ (соответственно $A$ на $A / M$ ) и $\omega: B / \mathscr{M} \rightarrow A / M-$ отображение, определяемое равенством $\omega\left[\pi_{B}(b)\right]=$ $=\pi_{A}\left[\Phi_{\varphi}(b)\right]$ при всех $b \in B$. Тогда из $b^{\prime}-b \in \mathcal{M}$ при всех $b^{\prime} \in \pi_{B}(b)$ следует, что $\pi_{A}\left[\Phi_{\varphi}\left(b^{\prime}\right)\right]=\pi_{A}\left[\Phi_{\varphi}(b)\right]$ при всех $b^{\prime} \in \pi_{B}(b)$. Поэтому отображение $\omega$ определено корректно. Нетрудно проверить, что $\omega$ является изоморфизмом $B / \mathcal{M}$ на $A / M$. В силу этого коразмерности идеалов $\mathcal{H}$ и $M$ одновременно либо равны единице, либо нет. Таким образом, из $\mathcal{M} \in \mathfrak{\mho}_{1}(B)$ следует $M \in \mathfrak{M}_{1}(A)$ и из $M \in \mathfrak{M}_{1}(A)$ следует $\mathcal{M} \in \mathfrak{\mho}_{1}(B)$. Поэтому отображение $(M, \varphi) \rightarrow \Phi_{\varphi}^{-1}(M)$ взаимно однозначно отображает $\mathfrak{M}_{1}(A) X$ hom $Z$ на $\mathfrak{J}_{1}(B)$ и $\mathfrak{M}_{1 c}(A) \times$ hom $Z$ на $\mathfrak{F}_{1 c}(B)$.

Доказательство для остальных случаев проводится аналогично.

\section{§ 2. Топологические модуль-алгебры, в которых нет свободных замкнутых максимальных идеалов коразмерности один}

В предыдущем параграфе дано описание фиксированных регулярных максимальных идеалов в топологических модуль-алгебрах. Возникает вопрос, в каких топологических модуль-алгебрах нет свободных максимальных идеалов? Следующая теорема дает ответ на этот вопрос в случае замкнутых максимальных идеалов, коразмерность которых равна единице. 
Т ео рем а 2. Пусть $A$ - отделимая топологическая F-алгебра, множество $\mathfrak{M}_{1 c}(A)$ которой непусто **, и $B$ - такая топологическая $(A, \mathbf{F})$ алгебра, которая содержит F-подалгебру $Z$, множество hom $Z$ которой непусто. Если выполнены условия (а), (б) и

в) $a z=z a$ при всех $a \in A u z \in Z$, то $\mathfrak{M}_{1 \mathrm{c}}(B)=\mathfrak{\mho}_{1 \mathrm{c}}(B)$.

Д ок а з а тельст в о. Пусть $\mathscr{M} \in \mathfrak{M}_{1 с}(B)$. Тогда $\mathscr{M}=\operatorname{ker} \Psi$ для некоторого гомоморфизма $\Psi \in$ hom $B$. По условию (а) существуют теперь элементы $a_{0} \in A$ и $z_{0} \in Z$ такие, что $\gamma=\Psi\left(a_{0} z_{0}\right) \neq 0$. Если это не так, то $\Psi(b)=0$ при всех $b$ из $A$-подмодуля $B_{Z}$ в $B$, порожденного F-подалгеброй $Z$. Поэтому по условию (а) было бы $\Psi(b) \equiv 0$ на $B$.

Пусть $\varphi=\Psi \mid Z$ и $\chi(a)=\gamma^{-1 \Psi}\left(a_{0} a z_{0}\right)$ для всех $a \in A$. Тогда по условию (в) справедливо

$$
\Psi\left(a_{0} a z_{0}\right)=\Psi\left[\left(a_{0} a z_{0}\right) a_{0} z_{0}\right] \gamma^{-1}=\gamma^{-1} \Psi\left[\left(a_{0} z_{0} a\right) a_{0} z_{0}\right]=\Psi\left(a a_{0} z_{0}\right)
$$

при всех $a \in A$. Поэтому при всех $a \in A$ имеет место равенство

$$
\Psi\left(a_{0} a z_{0}\right)=\Psi\left(a a_{0} z_{0}\right) .
$$

Если $a_{1} \in A$ и $z_{1} \in Z$ суть такие, что $\gamma_{1}=\Psi\left(a_{1} z_{1}\right) \neq 0$, то по равенству (1) и условию (в) имеем

$$
\Psi^{r}\left(a_{0} a z_{0}\right) \gamma_{1}=\Psi\left[\left(a_{0} a z_{0}\right) a_{1} z_{1}\right]=\Psi\left[\left(a_{0} z_{0} a\right) a_{1} z_{1}\right]=\psi^{\Psi}\left(a a_{1} z_{1}\right)=\Psi^{\prime}\left(a_{1} a z_{1}\right),
$$

откуда вытекает равенство

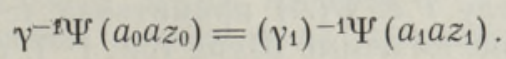

Таким образом, отображение $\chi$ не зависит от выбора элементов $a_{0}$ и $z_{0}$ и поэтому оно определено корректно.

Нетрудно заметить, что $\varphi$ есть непрерывный гомоморфизм $Z$ на $\mathbf{F}$, a $\chi$ - непрерывное линейное отображение $A$ на $\mathrm{F}$. Кроме того,

$$
\begin{aligned}
\chi\left(a_{1} a_{2}\right) & =\gamma^{-2} \Psi\left[a_{0} a_{1} a_{2} z_{0}\right] \gamma=\gamma^{-2} \Psi\left[\left(a_{0} a_{1} a_{2} z_{0}\right) a_{0} z_{0}\right]= \\
& =\gamma^{-2} \Psi\left[\left(a_{0} a_{1} z_{0}\right)\left(a_{2} a_{0} z_{0}\right)\right]=\chi\left(a_{1}\right) \gamma^{-1} \Psi\left(a_{2} a_{0} z_{0}\right)=\chi\left(a_{1}\right) \chi\left(a_{2}\right)
\end{aligned}
$$

при всех $a_{1}, a_{2} \in A$ по условию (в) и равенству (1). Поэтому $\chi$ есть непрерывный $\mathrm{F}$-гомоморфизм $A$ на $\mathrm{F}$. Поскольку

$$
\Psi(a z)=\gamma^{-1} \Psi\left[\left(a_{0} z_{0}\right) a z\right]=\gamma^{-1} \Psi\left[\left(a_{0} a z_{0}\right) z\right]=\chi(a) \varphi(z)
$$

при всех $a \in A$ и $z \in Z$ по условию (в), то из $\chi\left(a_{0}\right) \varphi\left(z_{0}\right)=\gamma$ следует, что гомоморфизмы $\chi$ и $\varphi$ нетривиальны. Следовательно, $\chi \in \operatorname{hom} A$ и $\varphi \in \operatorname{hom} Z$. (В частности, когда алгебра $B$ имеєт единицу, доказательство можно несколько упростить, так как отображение $\chi$, определяемое равенством $\chi(a)=\Psi\left(a e_{B}\right)$ при всех $a \in A$, является непрерывным F-гомоморфизмом на $A$ и справедливо $\Psi(a z)=\chi(a) \varphi(z)$ при всех $a \in A$ и $z \in Z$ не зависимо от того, выполнено условие (в) или нет. Таким образом, если алгебра $B$ имеет единицу, то условие (в) является лишним).

Пусть теперь $b-$ любой элемент в $B_{z}$. Тогда $b=a_{1} z_{1}+\ldots+a_{n} z_{n}$ для некоторых $n \in \mathbf{N}, a_{1}, \ldots, a_{n} \in A$ и $z_{1}, \ldots, z_{n} \in Z$. Пусть далее $\Phi_{\varphi} \in \operatorname{hom}(B, A)$ - гомоморфизм, определенный гомоморфизмом $\varphi$ по условию (б). Так как

$$
\Psi(b)=\sum_{k=1}^{n} \chi\left(a_{k}\right) \varphi\left(z_{k}\right)=\chi\left(\sum_{k=1}^{n} a_{k} \varphi\left(z_{k}\right)\right)=\chi \circ \Phi_{\varphi}(b),
$$

** Существуют топологические F-алгебры, в которых нет замкнутых максималыных идеалов (см. $\left[{ }^{4}\right]$, с. 125 или $\left[{ }^{3}\right]$, с. 146$)$. 
то отображения $\Psi$ и $\chi \circ \Phi_{\varphi}$ совпадают на $B$ по условию (а). В силу этого, $\Phi_{\varphi}(\mathcal{M}) \subset$ ker $\chi$. Следовательно, $\mathcal{M} \in \widetilde{\mho}_{1 c}(B)$.

3 амечание. В доказательстве теоремы 2 показано, что каждый $\Psi \in \operatorname{hom} B$ определяет $\chi \in \operatorname{hom} A$ и $\varphi \in \operatorname{hom} Z$ такие, что $\Psi=\chi \circ \Phi_{\varphi}$. Кроме того, $\chi \circ \Phi_{\varphi} \subset \operatorname{hom} B$ при всех $\chi \in \operatorname{hom} A$ и $\varphi \in \operatorname{hom} Z$. Далее из $\chi \neq \chi^{\prime} \quad\left(\varphi \neq \varphi^{\prime}\right)$ следует, что $\chi \circ \Phi_{\varphi}(a z) \neq \chi^{\prime} \circ \Phi_{\varphi^{\prime}}(a z)$ при $a \in$ $\in \operatorname{ker} \chi \backslash \operatorname{ker} \chi^{\prime}$ и $z \in Z \backslash\left(\operatorname{ker} \varphi \cup \operatorname{ker} \varphi^{\prime}\right) \quad($ соответственно при $a \in A \backslash$ $\backslash\left(\operatorname{ker} \chi \cup \operatorname{ker} \chi^{\prime}\right)$ и $\left.z \in \operatorname{kerr} \varphi \backslash \operatorname{ker} \varphi^{\prime}\right)$. Поэтому из $\chi \circ \Phi_{\varphi}=\chi^{\prime} \circ \Phi_{\varphi^{\prime}}$ следуют $\chi=\chi^{\prime}$ и $\varphi=\varphi^{\prime}$. Таким образом, справедлива

Т е о рем а 3. Пусть алгебры $A, B$ и Z суть такие, как в теореме 2. Если выполнены условия (а), (б) $и$ (в), то каждый $\Psi \in$ hom $B$ определяет $\chi \in \operatorname{hom} A$ и $\varphi \in \operatorname{hom} Z$ такие, что $\Psi=\chi \cdot \Phi_{\varphi}$ и отображение $(\chi, \varphi) \rightarrow$ $\rightarrow \chi \circ \Phi_{\varphi}$ является биекцией hom $A \times \operatorname{hom} Z$ на hom $B$.

В случае, когда $A$ и $B$ имеют единицу, теорема 3 известна (см. $\left.\left[{ }^{8}\right]\right)$. В этом случае условие (в) в теореме 3 является лишним.

\section{§ 3. Топологические модуль-алгебры с единицей, в которых нет свободных замкнутых максимальных идеалов}

Пусть $A$ - топологическая $\mathbf{F}$-алгебра, $\mathfrak{C}(A)$ - ее центр, $\mathfrak{B}(A)-$ множество всех ее примитивных идеалов (т. е. двусторонних идеалов в виде $\{a \in A: a A \in M\}$ и $\left\{a \in A: A a \in M^{\prime}\right\}$, где $M \in \mathbb{M}_{l}(A)$ и $M^{\prime} \in \mathbb{M}_{r}(A)$, a $\mathfrak{P}_{\mathrm{c}}(A)$ - подмножество замкнутых идеалов в $\mathfrak{P}(A)$. Тогда факторалгебра $A / P$ по идеалу $P \in \mathfrak{P}(A)$ примитивна (см. $\left[{ }^{9}\right]$, с. 136$)$ и ее центр 厄 $(A / P)$ есть поле по лемме Шура (см. $\left[{ }^{10}\right]$, теорема 1.1.1.). Таким образом, ᄃ $(A / P)$ (в зависимости от топологии алгебры $A$ ) может быть топологически изоморфным полю $\mathbf{F}$ или нет.

Чтобы выделить классы топологических $(A, \mathrm{~F})$-алгебр с единицей, в которых нет свободных замкнутых максимальных идеалов, нужна

Т е орем а 4. Пусть $A$ - отделимая топологическая F-алгебра с единицей, $B$ - топологическая $(A, \mathrm{~F})$-алгебра с единицей $e_{B}$ и $Z-$ подалгебра центра 巨( $(B)$, содержащая $e_{B}$. Если выполнены условия (а), (б’) (множество hom $Z$ непусто по условню (г) (см. доказательство теоремы)) и

(г) для каждого $\mathfrak{T} \in \mathfrak{P}_{\mathrm{c}}(B)$ поле $\mathfrak{C}(B / \mathfrak{T})$ является топологически изоморфным полю $\mathrm{F}$,

то $B$ не содержит свободных замкнутых максимальных идеалов.

Док а з а тельст в о. Пусть $\mathbb{M} \in \mathfrak{M}_{l c}(B)$ и $\mathfrak{T}=\{b \in B: b B \in \mathcal{M}\}$. Тогда $\mathfrak{S} \in \mathfrak{P}_{c}(B)$. Поэтому по условию (г) поля $\mathfrak{E}(B / \mathcal{T})$ и $\mathbf{F}$ топологически изоморфны. Этот изоморфизм обозначим через $\tau$. Пусть далее $x \rightarrow$ естественный гомоморфизм $B$ на $B / \mathfrak{T}, \quad x^{\prime}=x \mid x^{-1}(\mathbb{E}(B / \mathfrak{T}))$ и $\varphi=\left(\tau \circ x^{\prime}\right) \mid Z$. Тогда $\varphi \in \operatorname{hom} Z$. Поэтому по условию (б') существует $\Phi_{\varphi} \in \operatorname{hom}(B, A)$ такой, что $\Phi_{\varphi}(z)=e_{A} \varphi(z)$ для всех $z \in Z$. При этом

$$
\operatorname{ker} \Phi_{\varphi}=\mathrm{cl}_{B}(A \operatorname{ker} \varphi),
$$

где $\operatorname{cl}_{B} D$ обозначает замыкание подмножества $D \subset B$ в топологии алгебры $B$ и

$$
A \operatorname{ker} \varphi=\left\{\sum_{k=1}^{n} a_{k} z_{k}: n \in \mathbf{N} ; a_{1}, \ldots, a_{n} \in A \text { и } z_{1}, \ldots ; z_{n} \in Z\right\} .
$$

Чтобы показать это, берем любой элемент $b \in \operatorname{ker} \Phi_{\varphi}$ и любую окрестность нуля $O$ в $B$. Тогда $O_{1}+O_{1} \subset O$ при некоторой закругленной окрестности нуля $O_{1}$ в $B$ и (ввиду непрерывности отображения $\left.a \rightarrow a e_{B}\right)$ справедливо $V e_{B} \subseteq O_{1}$ при некоторой окрестности нуля $V$ в $A$. По условию 
(б’) гомоморфизм $\Phi_{\varphi}$ непрерывен. Поэтому в $B$ существует окрестность $\mathrm{O}_{2}$ такая, что $\Phi_{\varphi}\left(\mathrm{O}_{2}\right) \subseteq V$. Кроме того, по условию (а) существуют число $n \in \mathbf{N}$ и элементы $a_{1}, \ldots, a_{n} \in A$ и $z_{1}, \ldots, z_{n} \in Z$ такие, что

$$
c=\sum_{k=1}^{n} a_{k} z_{k}-b \in O_{1} \cap O_{2} .
$$

Положим $w_{k}=z_{k}-\varphi\left(z_{k}\right) e_{B}$ при всех $k=1$, $+a_{n} w_{n}$. Тогда $d \in A$ ker $\varphi$ и

$$
d-b=c-\Phi_{\varphi}(c) e_{B} \in O_{1} \cap O_{2}-V e_{B} \subset O_{1}+O_{1} \subset O .
$$

Поэтому $b \in K=\operatorname{cl}_{B}(A \operatorname{ker} \varphi)$. Следовательно, $\operatorname{ker} \Phi_{\varphi} \subseteq K$. Кроме того, справедливо включение $A \operatorname{ker} \varphi \subseteq \operatorname{ker} \Phi_{\varphi}$ по условию (б’). Так как множество $\operatorname{ker} \Phi_{\varphi}$ замкнуто в $B$, то справедливо и включение $K \subseteq \operatorname{ker} \Phi_{\varphi}$. Таким образом, показана справедливость равенства (2). Учитывая равенство (2), нетрудно заметить, что $\operatorname{ker} \Phi_{\varphi} \subseteq \mathcal{M}$ (ибо $\operatorname{ker} \varphi \subset \mathfrak{T} \subseteq \mathcal{M}$ ).

Пусть теперь $M=\Phi_{\varphi}(\mathcal{M})$. Тогда $M \neq A$ (если это не так, то $e_{A}=\Phi_{\varphi}(b)$ при некотором $b \in \mathcal{M}$, в силу чего было бы справедливо $e_{B}=$ $=b-\left(b-e_{B}\right) \in \mathcal{K}$, ввиду включения $\left.\operatorname{ker} \Phi_{\varphi} \subseteq \mathcal{M}\right)$. Поэтому $M$ образует в $A$ левый идеал.

Пусть далее $I$ - любой левый идеал в $A$, содержащий $M$. Тогда $\Phi_{\varphi}{ }^{-1}(I)$ образует в $B$ левый идеал, содержащий $\mathcal{H}$. Поэтому $\Phi_{\varphi}^{-1}(I)=\mathcal{M}$ ввиду максимальности идеала $\mathscr{M}$, и справедливо $I=M$. Таким образом, $M$ является максимальным левым идеалом в $A$. Чтобы показать его замкнутость, берем любой элемент $a_{0}$ из замыкания идеала $M$. Тогда в $M$ существует сеть $\left(a_{\lambda}\right)_{\lambda \in \Lambda}$, сходящаяся к $a_{0}$. Так как отображение $a \rightarrow e_{B} a$ непрерывно для каждого $a \in A$ и $e_{B} a_{\lambda} \in \mathcal{M}$ при всех $\lambda \in \Lambda$, то (ввиду замкнутости идеала $\mathcal{H}$ ) сеть $\left(e_{E} a_{\lambda}\right) \lambda \in \Lambda$ сходится к $e_{B} a_{0} \in \mathcal{M}$. Следовательно, элемент $a_{0}=\Phi_{\varphi}\left(e_{B} a_{0}\right) \in M$. В силу этого, $M \in \mathfrak{M}_{l c}(A)$ и справедливо $\mathscr{M} \in \widetilde{\mho}_{l c}(B)$.

Доказательство для правых и двусторонних идеалов проводится аналогично.

Оказывается, что во многих более изученных классах топологических C-алгебр условие (г) теоремы 4 выполнено. Действительно, справедлива

Те орем а 5. Пусть $A$ - отделимая топологическая С-алгебра с единицей, $B$ - одна из следующцх топологических C-алгебр

а) отделимая локально псевдовыпуклая $(A, \mathbf{C})$-алгебра с непрерывным обратным,

б) отделимая поглощценно выпуклая $(A, \mathbf{C})$-алгебра с единицей или

в) полная метризуемая локально выпуклая $(A, \mathbf{C})$-алгебра с единицей, а Z - F-подалгебра центра $匚(B)$, содержащая единицу алгебры $B$. Если выполнены условия (а) $и$ (б'), то В не содержит свободных замкнутых максимальных идеалов.

Доказ ательство. Учитывая теорему 4, достаточно показать только выполнение условия (г).

a) Пусть алгебра $B$ обладает свойством а) теоремы 5 и $\mathfrak{S} \in \mathfrak{P}_{c}(B)$. Тогда факторалгебра $B / \mathfrak{T}$ по идеалу $\mathfrak{S}$ есть отделимая локально псевдовыпуклая C-алгебра с непрерывным обратным (так как факторалгебра локально псевдовыпуклой $\mathbf{C}$-алгебры по двустороннему замкнутому идеалу локально псевдовыпукла и является отделимой топологической C-алгеброй с непрерывным обратным (см. $\left[{ }^{11}\right]$, с. 52$\left.)\right)$. Поэтому обра- 


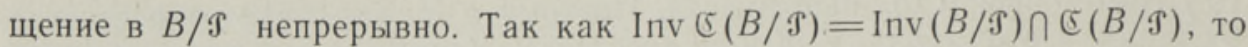
множество Inv $(B / \mathscr{T})$ открыто в $\mathfrak{C}(B / \mathscr{T})$ и обращение в $\mathfrak{5}(B / \mathfrak{T})$ непрерывно. Следовательно, С $(B / \mathrm{P})$ есть отделимое локально псевдовыпуклое поле над $\mathbf{C}$ с непрерывным обратным и поэтому является тотологически изоморфным полю С (см $\left[{ }^{12}\right]$, с 153$)$.

б) Пусть алгебра $B$ обладает свойством б) теоремы 5 и $\left\{p_{\beta}: \beta \in \mathrm{B}\right\}$ - система непрерывных полунорм на $B$, определяющая ее топологию. Пусть далее $\mathfrak{T} \in \mathfrak{B}_{c}(B), \pi$ обозначает естественный гомоморфизм $B$ на $B / \mathfrak{T}$ и пусть

$$
q_{\beta}(\pi(b))=\inf \left\{p_{\beta}\left(b^{\prime}\right): b^{\prime} \in \pi(b)\right\}
$$

для всех $\beta \in \mathrm{B}$ и $b \in B$. Тогда система полунорм $\left\{q_{\beta}: \beta \in \mathrm{B}\right\}$ определяет на $B / \mathfrak{T}$ локально выпуклую топологию. Нетрудно заметить, что эта топология на $B$ поглощенно выпукла. Поэтому центр $\mathfrak{E}(B / \mathfrak{T})$ есть отделимое поглощенно выпуклое поле над $\mathbf{C}$ с единицей, в силу чего, поля ᄃ $(B / \mathfrak{T})$ и С топологически изоморфны (см. $\left[{ }^{13}\right]$, с. 474$)$.

в) Пусть в заключение алгебра $B$ обладает свойством в) теоремы 5 и $\mathfrak{T} \in \mathfrak{F}_{c}(B)$. Тогда факторалгебра $B / \mathfrak{T}$ по идеалу $\mathfrak{T}$ является полной метризуемой локально выпуклой С-алгеброй с единицей. Поскольку центр топологической алгебры замкнут, то $\Subset(B / S)$ есть полное метризуемое локально выпуклое поле над $\mathbf{C}$ с единицей. Таким образом, поля ᄃ $(B / \mathcal{T})$ и С топологически нзоморфны (см. [ $\left.{ }^{4}\right]$, с. 86).

3 а меч ан ие. Как отмечено выше, каждая локально выпуклая C-алгебра и каждая локально ограниченная C-алгебра локально псевдовыпуклы, причем обращение в отделимой полной локально ограниченной C-алгебре с единицей $A$ непрерывно и множество Inv $A$ открыто (см. $\left[{ }^{4}\right]$, c. $17-18)$. Поэтому отделимые полные локально ограниченные C-алгебры являются отделимыми локально псевдовыпуклыми C-алгебрами с непрерывным обратным. Кроме того, каждая отделимая локально $m$-выпуклая C-алгебра (в частности, нормированная в банахова С-алгебра) поглощенно выпукла. Таким образом, по теореме 5 отделимые полные локально ограниченные $(A, \mathbf{C})$-алгебры с единицей, отделимые локально выпуклые $(A, \mathbf{C})$-алгебры с непрерывным обратным и отделимые локально $m$-выпуклые $(A, \mathrm{C})$-алгебры (в частности, нормированные и банаховы $(A, \mathbf{C})$-алгебры) с единицей не содержат свободных замкнутых максимальных идеалов, если алгебры $A$ и $Z$ суть такие же как в теореме 5 и выполнены условия (а) и (б'). При этом естественно, что вышеописанные ограничєния относительно алгебры $B$ влекут за собой и свои дополнительные ограничения относительно алгебры $A$.

\section{$\S$ 4. Топологические модуль-алгебры с единицей, в которых нет свободных идеалов}

Пусть $A$ - топслогическая $\mathrm{F}$-алгебра с единицей, a $\operatorname{Inv}_{l} A$ и $\operatorname{Inv}_{r} A-$ множества всех элементов алгебры $A$, имеющих, соответственно, левый обратный и правый обратный. Пусть далее $X$ - топологическое пространство и $C(X, A)$ - F-алгебра (относительно поточечных алгебраических операций) всех $A$-значных непрерывных отображений на $X$, наделенная топологией равномерной сходимости. Нетрудно заметить, что отображение $e$ с $e(x) \equiv e_{A}$ является единицей алгебры $C(X, A)$.

Следующая теорема определяет класс топологических модуль-алгебр с единицей, все идеалы которых фиксированы.

T ео рем а 6. Пусть $A$ - отделимая топологическая F-алгебра с непрерывным обратным и $B$ - топологическая $(A, \mathrm{~F})$-алгебра с единицей $e_{B}$, 
содержащая F-подалгебру $Z$ такую, что $e_{B} \in Z$ и пространство hom $Z$ непусто и компактно. Если выполнены условия (а), (б'),

(д) $\mathcal{A}(B)$ является всюду плотным в $C$ (hom $Z, A) u$

(e) $и з \quad b \in B u b^{\wedge} \in C\left(\operatorname{hom} Z, \operatorname{Inv}_{k} A\right)$ следует $b \in \operatorname{Inv}_{k} B$ nрu $k=l, r$, то все идеалы в В фиксированы.

Доказ ательство. Пусть $\mathfrak{J}$ - любой левый идеал в $B$. Если $\mathfrak{J}$ свободен, то для каждого $\varphi \in \operatorname{hom} Z$ существует $b_{\varphi} \in \mathcal{J}$ такой, что $\left(b_{\varphi}\right)^{\wedge}(\varphi) \in \operatorname{Inv}_{l} A$. Поскольку $A$ есть топологическая $\mathbf{F}$-алгебра с непрерывным обратным, то множество $\operatorname{Inv}_{l} A$ открыто в $A$ и левостороннее обращение $a \rightarrow a_{l}{ }^{-1}$ является непрерывным отображением $\operatorname{Inv}_{l} A$ в $\operatorname{Inv}_{l} A$ (см. $\left[{ }^{1}\right]$, c. 205). Кроме того, отображения $\left(b_{\varphi}\right)^{\wedge}$ непрерывны на hom $Z$ для каждого $b \in B$ по условию (д). Поэтому для каждого $\varphi \in$ hom $Z$ существует окрестность $O_{\varphi}$ такая, что $\left(b_{\varphi}\right)^{\wedge}(\psi) \in \operatorname{Inv}_{l} A$ при всех $\psi \in O_{\varphi}$. Ввиду регулярности пространства hom $Z$, существуют такие открытые окрестности $U_{\varphi}$, что $\varphi \in U_{\varphi} \subset \mathrm{cl}_{\text {homZ }} U_{\varphi} \subset O_{\varphi}$. Таким образом, множества $U_{\varphi}$ с $\varphi \in$ hom $Z$ образуют открытое покрытие компактного пространства hom $Z$. Следовательно, найдется конечное число множеств $U_{\varphi(1)}, \ldots, U_{\varphi(n)}^{t}$, которые покрывают hom $Z$. Теперь существует разбиение единицы $\alpha_{1}, \ldots, \alpha_{n} \in C$ (hom $\left.Z,[0,1]\right)$, определяемое множествами $U_{\varphi(1)}, \ldots, U_{\varphi(n)}$ (см. $\left[{ }^{1}\right]$, с. 260$)$, причем $\alpha_{k}(\varphi)=0$ при $\varphi \notin U_{\varphi(k)}$ и $\alpha_{1}(\varphi)+\ldots+\alpha_{n}(\varphi) \equiv 1$ на hom $Z$.

Пусть $g_{k}$ - отображения hom $Z$ в $A$ такие, что

$$
g_{k}(\varphi)=\left\{\begin{array}{lll}
\left.\alpha_{k}(\varphi)\left(b_{\varphi(k)}\right)^{\wedge}(\varphi)\right)_{l}^{-1} & \text { при } & \varphi \in \operatorname{cl}_{\text {hom } Z} U_{\varphi(k)} \\
\theta_{A} & \text { при } & \varphi \in \operatorname{hom} Z \backslash U_{\varphi(k)}
\end{array}\right.
$$

для всех $k=1, \ldots, n$, где $\theta_{A}-$ нулевой элемент в $A$. Поскольку левосторонее обращение в $A$ непрерывно и $g_{k}(\varphi)=\theta_{A}$ на $\left(\operatorname{cl}_{\text {hom Z }} U_{\varphi(k)}\right) \backslash$ $\backslash U_{\varphi(k)}$, то $g_{k} \in C(\operatorname{hom} Z, A)$ при всех $k$ (см. $\left[{ }^{14}\right]$, с. 26$)$ и справедливо

$$
\operatorname{Inv}_{l} C(\operatorname{hom} Z, A)=C\left(\operatorname{hom} Z, \operatorname{Inv}_{l} A\right) \text {. }
$$

Как отмечено выше, множество $\operatorname{Inv}_{l} A$ открыто в $A$ : Поэтому множество $W=\operatorname{Inv}_{l} C($ hom $Z, A)$ открыто в $C(\operatorname{hom} Z, A)$, в силу чего, $W$ есть окрестность единицы $e$ в $C$ (hom $Z, A)$.

Пусть теперь $W=e+O$ для некоторой окрестности нуля $O$ в $C(\operatorname{hom} Z, A)$, а $O_{1}, \ldots, O_{n}, U_{1}, \ldots, U_{n}-$ окрестности нуля в $C(\operatorname{hom} Z, A)$ такие, что $O_{1}+\ldots+O_{n} \subset O$ и $U_{k}\left(b_{\varphi(k)}\right) \wedge \subset O_{k}$ при всех $k$. Тогда по условию (д) для каждого $k$ существует элемент $b_{k} \in B$ такой, что $\left(b_{k}\right)^{\wedge}-g_{k} \in U_{k}$. Положив $c=b_{1} b_{\varphi(1)}+\cdots+b_{n} b_{\varphi(n)}$, заметим, что $c \in \mathfrak{J}$ и справедливо

$$
\hat{c}=\sum_{k=1}^{n}\left[\left(b_{k}\right)^{\wedge}-g_{k}\right]\left(b_{\varphi(k)}\right) \hat{}+\sum_{k=1}^{n} g_{k}\left(b_{\varphi(k)}\right)^{\wedge} \in W .
$$

Но это не возможно по условию (е). Таким образом, $B$ не содержит свободных идеалов, если она удовлетворяет условиям (а), (б’), (д) и (е). Доказательство для правых идеалов. проводится аналогично.

3 а м е ч а и е. Оказывается, что условие (е) теоремы 6 выполнено, если

1) $t$ является биекцией $B$ на $C(\operatorname{hom} Z, A)$,

2) $B$ есть отделимая локально псевдовыпуклая $(A, \mathbf{C})$-алгебра с непрерывным обратным и 
3) $B$ есть отделимая полная локально $m$-выпуклая коммутативная $(A, \mathbf{C})$-алгебра с единицей.

В самом деле, из утверждения 1) непосредственно следует выполнение условия (е). Кроме того, если выполнены утверждения 2) или 3) и $b^{\wedge} \in C\left(\operatorname{hom} Z, \quad \operatorname{Inv}_{k} A\right)$, то $b^{\wedge}(\varphi) \notin \cup\left\{M: M \in \mathfrak{M}_{k}(A)\right\}$ при всех $\varphi \in$ $\in \operatorname{hom} Z, \quad$ где $k=l, r$. Поэтому $b^{\wedge}(\varphi) \notin M$ для каждых $\varphi \in \operatorname{hom} Z$ и $M \in \mathfrak{M}_{k c}(A)$. Таким образом, справедливо $b \notin U\left\{\mathcal{M}: \mathcal{M} \in \mathfrak{M}_{k c}(B)\right\}$ при $k=l, r$ по теореме 5 . Поскольку в первом случае все максимальные идеалы в $B$ замкнуты, а во втором случае имеет место равенство

$$
\cup\{\mathscr{M}: \mathscr{M} \in \mathfrak{M}(B)\}=\bigcup\left\{\mathscr{M}: \mathcal{M} \in \mathfrak{M}_{c}(B)\right\}
$$

(см. $\left[{ }^{15}\right]$, с. 231 ), то отсюда вытекает, что $b \in \operatorname{Inv}_{k} B$.

В заключение отметим, что примеры топологических модуль-алгебр, которые имеют свободные идеалы, приведены в [ [ $\left.{ }^{16}\right]$ на с. 369 и 371.

\section{Л И Т Е Р А Т У Р А}

1. Наймарк М. А. Нормированные кольца. М. «Наука», 1968.

2. Cochran, A. C., Krown, R., Williams, C. R. Pacif. J. Math., 34, 17-25 (1970).

3. Khaleelulla, S. M. Counterexamples in Topological Vector Spaces. Lect. Notes in Math., 936. Berlin, Springer-Verlag, 1982.

4. Zelazko, W. Lect. Notes Math., № 31 (1971).

5. Абель М. А. В кн.: Тез. докл. VI Прибалтийской конференции по современным проблемам геометрии и их применениям. Таллин, 1984, 3-4.

6. Zelazko, W. Rozpr. Mat., XLVII, (1965).

7. Simmons, G. F. Introduction to Topology and Modern Analysis. New York, McGrow-Hill, 1963.

8. Abel, M. In: Abstracts. Colloquim on Topology, Eger, Aug. 9-13, 1983. Budapest, Janos Bolyai Math., Soc., 1983, 2.

9. Bonsall, F. F., Duncan, J. Complete Normed Algebras. Berlin, Springer-Verlag, 1973.

10. Херстейн И. Некоммутативные кольца. М., «Мир», 1972.

11. Waelbroeck, $L$. Theorie des algèbres de Banach et des algèbres localement convexes. Univ. de Montréal, 1962.

12. Waelbroeck, L. Continuous Inverse Locally Pseudoconvex Algebras. Summer school on topological algebra theory, Sept. 6-16, 1966. Bruges, 1966, 128-185.

13. Cochran, A. C. Proc. Amer. Math. Soc., 41, № 2, 473-479 (1973).

14. Александров П. С., Пасынков Б. А. Введение в теорию размерности. М., «Наука», 1973.

15. Beckenstein, E., Narici, L., Suffel, Ch. Topological Algebras. North-Holland Math. Studies, 24. Amsterdam, North-Holland Publ. Company, 1977.

16. Hery, W. J. Pacif. J. Math., 65, 365-373 (1976).

Тартуский государственный университет
Поступила в редакцию $21 /$ VI 1984

M. $A B E L$

\section{FIKSEERITUD IDEAALID TOPOLOOGILISTES MOODUL-ALGEBRATES}

On antud fikseeritud regulaarsete maksimaalsete ideaalide kirjeldus topoloogilistes moodul-algebrates ning kirjeldatud topoloogiliste moodul-algebrate klasse, milledes on fikseeritud kas

1) kõik regulaarsed kinnised maksimaalsed ideaalid, millede kodimensioon on 1; või

2) kõik kinnised maksimaalsed ideaalid

3) kōik ideaalid. 


\section{FIXED IDEALS IN TOPOLOGICAL MODULE-ALGEBRAS}

Let $\mathbf{F}$ be one of the fields $\mathbf{R}$ or $\mathbf{C}, A$ be a topological $\mathbf{F}$-algebra and hom $A$ be the set of all non-zero continuous $\mathbf{F}$-homomorphisms of $A$ onto $\mathbf{F}$. Let $B$ be a topological module-algebra with respect to $A$, or, in short, a topological $(A, \mathbf{F})$-algebra (that is a topological F-algebra which at the same time is a $A$-bimodule with separately continuous module multiplications and in which the conditions $a\left(b_{1} b_{2}\right)=\left(a b_{1}\right) b_{2}, \quad\left(b_{1} b_{2}\right) a=$ $=b_{1}\left(b_{2} a\right)$ and $\left(b_{1} a\right) b_{2}=b_{1}\left(a b_{2}\right)$ hold for each $a \in A$ and $\left.b_{1}, b_{2} \in B\right)$ which contains such a F-subalgebra $Z$ that the set hom $Z$ is not empty. Moreover, let $B$ satisfy the following conditions:

(a) the A-submodule of $B$ generated by $Z$ is dense in $B$ and

(b) for each $\varphi \in$ hom $Z$ there exists a non-zero continuous A-homomorphism $\Phi_{\varphi}$ of $B$ onto $A$ such that $\Phi_{\varphi}(a z)=\Phi_{\varphi}(z a)=a \varphi(z)$ for all $a \in A$ and $z \in Z$.

The left (right and two-sided) ideal $J$ of $B$ we shall call fixed if there exists a left (respectively, right and two-sided) ideal $I$ of $A$ such that $\Phi_{\varphi}(\mathcal{J}) \subseteq I$.

In the present paper the descriptions of all fixed regular maximal (in particular, maximal closed) ideals and all such fixed maximal ideals, codimension of which is one, are given. Moreover, the classes of topological module-algebras in which are fixed a) all regular maximal closed ideals with codimensions one, and

b) all closed maximal ideals

c) all ideals

are described. 